\title{
RESGATE DA CIDADANIA E DA CULTURA DE PAZ NA TERAPIA DO REENCONTRO MEDIADO
}

\author{
Janete Rosa Martins ${ }^{1}$ \\ Francéli Raquel Radons ${ }^{2}$
}

\begin{abstract}
RESUMO: O presente tem como finalidade analisar a terapia do reencontro mediado como resgate da cidadania e cultura da paz. Modelo esse, proposto por Luiz Alberto Warat como uma concepção transformadora da mediação, construída para fomentar a autonomia, emancipação dos sujeitos numa relação conflitiva, um novo paradigma de autoconhecimento. A metodologia utilizada e o hipotético dedutivo, como técnica de pesquisa indireta em doutrinas. A discussão e aprofundamento se dá pelos conceitos da teoria do reencontro mediado proposto com o resgate da cidadania e da cultura de paz. Tema abrangente e se justifica pelo debate da mediação na teoria do reencontro mediado.
\end{abstract}

Palavras-chave: teoria do reencontro mediado - conflitos - cidadania - cultura de paz

\section{RESCUE OF CITIZENSHIP AND PEACE CULTURE IN THERAPY OF THE MIDDLE REINCONTRY}

\begin{abstract}
The purpose of this paper is to analyze the therapy of mediated reunion as a rescue of citizenship and a culture of peace. This model, proposed by Luiz Alberto Warat as a transformative conception of mediation, built to foster autonomy, emancipation of subjects in a conflictive relationship, a new paradigm of self-knowledge. The methodology used and the hypothetical deductive, as an indirect research technique in doctrines. The discussion and deepening is given by the concepts of the theory of mediated reunion proposed with the rescue of citizenship and the culture of peace. Broad subject and justified by the debate of mediation in the theory of mediated reunion.
\end{abstract}

Keywords: mediated reunification theory - conflicts - citizenship - culture of peace

\footnotetext{
${ }^{1}$ Doutora em Ciências Sociais - UNISINOS - São Leopoldo/RS, Mestre em Direito - UNISC - Santa Cruz do Sul/RS Especialista em Direito Público e graduada em Direito - UNIJUI - Ijuí/RS, Professora Programa de PósGraduação Stricto Sensu em Direito - Mestrado e Doutorado, graduação (URI), campus de Santo Ângelo, e-mail janete@san.uri.br.

${ }^{2}$ Mestranda em Direitos Especiais pela Universidade Regional Integrada do Alto Uruguai e das Missões (URI) Santo Ângelo. E-mail: franceli.radons@hotmail.com.
} 


\section{INTRODUÇÃO}

O presente artigo tem como finalidade analisar a terapia do reencontro mediado como resgate da cidadania e cultura da paz. Modelo esse proposto por Luiz Alberto Warat em uma concepção transformadora da mediação, construída para fomentar a autonomia e emancipação dos sujeitos numa relação conflitiva, como um novo paradigma de autoconhecimento. Diante disso questiona-se: De que maneira a terapia do reencontro mediado resgata a cidadania e a cultura da paz em uma relação conflitiva entre os atores envolvidos? O método de abordagem é o hipotético dedutivo, com técnica de pesquisa indireta em literaturas de Luis Alberto Warat e outros.

Além do mais, a sociedade brasileira encontra-se mergulhada em impasses e desafios, em direção a uma sociedade capaz de atender as necessidades fundamentais dos cidadãos, especialmente quanto à efetividade da cidadania. O tema é de abrangência e se justifica pelo debate em torno da mediação e suas especificidades para a resolução de conflitos.

\section{O CONFLITO E A MEDIAÇÃO}

Ao longo da vida o ser humano busca satisfazer suas motivações, estabelecendo relações afetivas, profissionais e sociais com outras pessoas, as quais também buscam satisfazer as suas motivações. Assim, quando o comunicador e o receptor divergem em suas construções mentais, conceitos, preconceitos e intenções, ou seja, quando o diálogo é interrompido, estamos diante de um conflito.

Na história da humanidade as mudanças vieram sempre após algum conflito, seja por guerras ou individuais, mas sempre tem pessoas envolvidas as quais são responsáveis pelos acontecimentos. Disso, pode-se falar que a vida é um conflito, é movimento, é crescimento, é desenvolvimento (SILVA, 2004, p. 91-92).

Os conflitos são inerentes a vida humana, é perceptível que as pessoas são diferentes, tanto em descrições pessoais, quanto em pontos de vista distintos, muitas vezes colidentes. Instalando-se fortes perturbações na paz social, logo, remetendo a insatisfação das pessoas quando seus interesses divergem.

Nas palavras de Luis Alberto Warat, o conflito é assim compreendido e analisado como uma estrutura funcional comunicativa que gera significados que devem ser interpretados como as bases da disputa (2010, p. 15). 


\section{RESGATE DA CIDADANIA E DA CULTURA DE PAZ NA TERAPIA \\ DO REENCONTRO MEDIADO}

Nessa linha, o conflito e a insatisfação tornam-se necessários para o aprimoramento das relações interpessoais e sociais. O que reflete algo bom ou ruim para as pessoas é a administração do conflito (SALES, 2007, p. 23-24).

Em regra, o conflito está presente em qualquer contexto das relações interpessoais e sociais e normalmente é compreendido como algo ruim, um fenômeno negativo. Com a visão negativa, o conflito é visto como uma ameaça e conseqüente reação de luta e de fuga, desenvolvendo um espiral de condutas conflitivas, onde ambas as partes são vítimas, ofensores e atores do ato.

Dessa forma, o rompimento e o enfraquecimento das relações sociais passam a ser inevitáveis, o sentimento de angustia, hostilidade, disputa, agressões, tristeza e tantos outros mais, passam a envolver as partes eliminando sua qualidade de vida e conseqüente a pacificação social.

Em contrapartida, quando se percebe que um impasse pode ser um momento de reflexão e, em conseqüência, de transformação, torna-se algo de positivo (SALES, 2007, p. 24).

Nas palavras de Silva:

[...] Note-se que a idéia de conflito atrelada às coisas ruins e destrutivas, na verdade não é uma visão adequada, visto que todo o crescimento, todo o conhecimento, toda a evolução e até a própria vida estão ligados ao conceito de conflito, por isso devemos ter uma visão positiva do conflito, como uma oportunidade de crescimento, de aprendizado de vida (2004, p. 93).

Logo, o conflito não é algo que deva ser encarado negativamente, pois cada pessoa é dotada de uma originalidade, personalidade única, por mais que exista afeto e afinidade nas relações interpessoais, algum conflito estará presente e essa consciência conflitiva é inerente à condição humana.

Em geral, o conflito é encarado de forma negativa, transformando-se em um verdadeiro tormento para as partes, pois o que se busca é o fim da controvérsia a qualquer custo, não importando as pessoas em si. E ao longo da evolução da sociedade o Estado por meio do judiciário promoveu através de um Juiz a intervenção nos conflitos entre duas ou mais pessoas, impondo-lhes uma solução conforme a lei.

E sob esse pensamento, floresce a desesperança, a falta de autoestima, as diferenças e a desconfiança em tudo e em todos, pois quando se decide judicialmente, consideram-se normativamente os efeitos; deste modo o conflito pode ficar hibernado, retornando agravado em qualquer momento futuro (WARAT, 2010, p. 09). 
Logo, os conflitos interpessoais envolvem sentimentos, afetos e valores, pois se funda em uma expectativa, nesse sentido, o judiciário vem mostrando-se incapaz de solucionar de forma satisfatória os dilemas e controvérsias quotidianas, pois impõe soluções aparentes, porém de forma subjacente atua como intensificador das angustias, insatisfações, intranqüilidades e mal-estares.

Partindo dessa ótica, o conflito em si não é o problema, o problema é a forma de lidar com ele, por isso deve ser encarado de forma positiva, transformadora que vislumbra o crescimento, o aprendizado e a melhora na qualidade de vida das partes.

Com essa concepção afirma Warat:

\begin{abstract}
A orientação transformadora vê o conflito como uma das principais forças positivas na construção das relações sociais e na realização da autonomia individual. A diferença de força puramente negativa, autodestrutiva da indiferença, o conflito brinda com um incentivo para a interação, termina erigindo-se numa possibilidade para criar, com o outro, a diferença (WARAT, 1998, p. 16).
\end{abstract}

O caminho para alcançar a transformação deste conflito depende do reconhecimento das diferenças, dos interesses comuns e do contraditório das partes. Toda pessoa é causa do comportamento de outra pessoa, e esta por sua vez, é comportamento daquela.

Assim, o conflito quando bem conduzido, é fonte geradora de mudanças, nascendo oportunidades de crescimento, pois o que propõe uma gestão positiva do conflito é justamente a força potente do conflito, uma vez apropriando-se desta força, se constrói novas realidades mais benéficas para todos os envolvidos na disputa.

Como bem refere Warat, todo conflito obriga a uma situação de influências recíprocas que têm que ser resgatadas em suas potencialidades, para produzir a diferença na disputa e em cada uma das perspectivas de vida das partes enfrentadas (1998, p. 16).

A partir de uma visão positiva do conflito, o trabalho de decidir quem está certo e quem está errado, deixa de existir e passa a se desenvolver a construção de soluções por meio da participação de todos os envolvidos na controvérsia, as pessoas passam a compreender a sua autonomia e passam a tomar posse dela para gerir suas decisões.

O instituto da mediação é um sistema inteligente de solução, resolução e administração de conflitos, primeiro, porque é voltado para a satisfação de ambas as partes, e segundo porque 


\section{RESGATE DA CIDADANIA E DA CULTURA DE PAZ NA TERAPIA \\ DO REENCONTRO MEDIADO}

promove a estrutura de um diálogo que possibilita uma comunicação acurada, além de acelerar a solução em termos eficazes de qualidade, tempo e aplicabilidade.

Dessa forma, com a prática da mediação se estabelece a participação ativa das pessoas nas soluções de conflitos, passa-se a não somente se discutir sobre questões individuais, mas questões de natureza coletiva também (SALES, 2007, p. 38).

Pode-se dizer que a mediação contribui com a comunicação maleável num momento de necessidade de resgatar legislações condizentes para a sociedade contemporânea, daí a necessidade de considerar a dimensão do diálogo voltada para o coletivo participativo. Pois, por meio da mediação se propõe um viés integrativo aonde o povo e legislador ocupam lugares igualitários, lado a lado.

Dessa forma, a mediação é um caminho que propõe o protagonismo das partes envolvidas no conflito, demonstra a importância do (re) encontro entre elas, institui a possibilidade do diálogo, atua na transformação do conflito e das pessoas, valida a autonomia, onde as quais passam a construir por si só, uma decisão mais amorosa a partir da percepção do outro, reencontrando-se em suas pulsões de vida.

Na mediação importa trabalhar o conflito sobre a diferença do outro, realizando a sensibilidade na forma de atingir a simplicidade do conflito e a realidade que o envolve, de forma que a justiça seja construída em conjunto pelos envolvidos no conflito, proporcionando autonomia, melhor qualidade de vida, aprimorando os sentimentos e conseguinte pacificação social.

Em uma acepção ampla, autonomia faz referência à capacidade humana de autodeterminação sobre sua própria vida e com os outros. Luis Alberto Warat, por sua vez, expressa:

[...] a idéia de autonomia aparece referida a necessidade de que o homem não aceite ser condicionado por regras que ele mesmo não possa determinar em função dos fins que ele próprio se propõe ou dos fins que institui em uma comunicação não alienada com os outros (2004, p. 328).

Nessa perspectiva, a autonomia não é algo isolado, é sempre aprendida com o outro. Um ser humano só pode ser autônomo na medida em que reconhece e garante a autonomia dos outros. Falar de autonomia, [...] é se ocupar da capacidade das pessoas para se auto 
determinarem em relação e com os outros; autodeterminarem-se na produção da diferença (WARAT, 1998, p. 07).

Na contemporaneidade a autonomia tem sido centrada na noção de individualidade, a partir da indiferença pelo outro, aproximando-se cada vez mais de uma concepção de alienação.

Nesse teor, para que exista autonomia e reconhecimento das diferenças, teremos que aceitar o caráter inacabado e indeterminável das relações sociais, dado que elas, em cada instante, se refazem de um modo imprevisível (WARAT, 2004, p. 328).

Dentro dessa expectativa, é imprescindível que se renuncie ao mito de uma sociedade perfeita, a qual se revela pautada nos ideais de competitividade e individualismo, para que então, se possa existir a autonomia e o reconhecimento das diferenças.

Para tanto, Warat trata a autonomia como uma forma de produzir diferenças e tomar decisões com relação ao conflito determina e nos configura, em termos de identidade e cidadania (WARAT, 1999, p. 07).

Sob essa ótica, o processo de mediação se apresenta como um meio de tratamento de conflitos não adversarial, logo, é uma forma ecolológica que aponta a uma melhor qualidade de vida as pessoas envolvidas em um conflito.

A tarefa do reconhecer-se como absolutamente responsável pelas suas decisões e escolhas bem como pelas suas ações é desafiadora e, por isso, a mediação oferece aos cidadãos participação ativa no tratamento de seus anseios, resultando no crescimento do sentimento de responsabilidade, de cidadania e de controle sobre os problemas vivenciados.

A mediação tem em si um caráter pedagógico e deve ser encarada como uma atitude geral diante da vida, como uma visão de mundo, um paradigma ecológico e um critério epistêmico de sentido (WARAT, 2010, p. 05).

Assim, a satisfação substitui a aplicação coercitiva de uma sanção legal, permitindo que as próprias partes priorizem o diálogo como principal meio de transformação dos conflitos de modo a realizar sua autonomia para a tomada de decisões com relação à conflitividade.

No olhar de Luis Alberto Warat,

“É importante considerar que as práticas da mediação se configuram em um instrumento de realização da autonomia, da democracia e da cidadania, na medida em que educam, facilitam e ajudam a produzir diferenças e a realizar tomadas de decisões sem a intervenção de terceiros que decidam pelos afetados por um conflito” (1998, p. 06). 
Ainda nas palavras do autor,

\begin{abstract}
"a autonomia pode ser pensada como uma possibilidade de escapar do mundo das palavras e dos mitos que nos exilam do real. A vontade persistente de toda República [...] é a de manter os cidadãos felizes como dependentes incuráveis das palavras e dos mitos. O cidadão que não pensa, porém, acredita que pensa, repetindo chavões. Escuta falar que é livre e acredita nas palavras, enquanto a vida lhe grita, e ele não escuta, que é um prisioneiro de sua própria imbecilidade energizada pelas palavras e pelos mitos" (WARAT, 2004, p. 15).
\end{abstract}

De acordo com Lília Maia de Morais Sales, a mediação apresenta-se como uma forma amigável e colaborativa de solução de controvérsias que busca a melhor solução pelas próprias partes (2004, p. 23).

E a autora continua, as partes, no processo de mediação, detêm a gestão de seus conflitos e, consequentemente, o poder de decidir, tendo o mediador como auxiliar, diferentemente da jurisdição estatal (2004, p. 24).

A partir disso, a mediação representa um mecanismo de tratamento de conflitos com a participação ativa das pessoas por meio da comunicação e para que esse objetivo seja alcançado o caminho é o diálogo com autonomia. Nessa perspectiva, a autonomia da vontade dos indivíduos é essencial para a proveitosa transformação dos conflitos.

“[...] a mediação pressupõe a transformação dos conflitos por um trabalho que aponte para a realização da autonomia das partes envolvidas no conflito. A autonomia de cada um, como a produção com o outro do novo (diferença). A autonomia como possibilidade de me transformar olhando-me a partir do olhar do outro” (WARAT, 1998, p. 08).

Por meio do diálogo e do empoderamento das partes se estimula a transformação conjunta delas, pois passam a compreender suas responsabilidades no ato conflitivo e assim, assumindo uma posição ativa em relação à solução, a qual é construída com autonomia e cooperação dos conflitantes.

Nesse panorama, o mecanismo da mediação, restabelece os laços rompidos e através dela é conferida autonomia às partes, que constroem conjuntamente, por si só a solução do anseio compartilhado. Permitindo assim, um acesso e um procedimento mais democrático, pacífico e humano, restabelecendo vínculos e uma melhor qualidade de vida as pessoas.

Enquanto no meio tradicional de solução de controvérsias o processo é abordado como antagonista, apontando as partes como inimigas, como ganhador e perdedor, como certo e 
errado, sendo imposta uma decisão pelo Estado-Juiz. No processo de mediação, o conflito é visto como forma de crescimento, busca do diálogo, auto-reflexão, ambas as partes são ganhadoras (SALES, 2004, P. 65).

Portanto, a autonomia da vontade na mediação reflete aos próprios envolvidos no ato conflitivo a decisão, se participarão da mediação, se querem conversar, se querem construir uma solução consensual, se querem interromper a sessão ou até mesmo desistir dela. Desse modo, as partes têm ampla autonomia.

Cumpre frisar que a autonomia está vinculada, sem dúvida, ao conceito de liberdade e por isso somente os indivíduos que estão vivenciando o problema são responsáveis por uma possível solução, mas não um acordo de palavra, os quais são fracos, falsos e correm o risco de agravar o conflito e sim acordos do coração, promessas assinadas desde os sentimentos.

Nas palavras de Warat:

Quando o acordo é de palavras, seu cumprimento sempre será altamente duvidoso com as promessas de amor feitas em nome do futuro. Nos processos de mediação, como negociação transformadora, em regra geral, se procura chegar a um acordo”. de palavras, a um acordo que se celebra desde a mente. Esses acordos são fracos, falsos, correm o risco de agravar o conflito. O mediador tem que ajudar as partes para que possam celebrar acordos do coração, promessas assinadas desde os sentimentos, sentidas, totais. Ele deve evitar que as partes prometam unicamente com a sua parte mental ou algum tipo de interesse, que façam um acordo de pensamentos, pois esse compromisso faz nascer a hipocrisia (2004, p. 30).

Entretanto o acordo pode vir ou não, nessa perspectiva, ao se conseguir facilitar a conversa, conscientizando as partes de sua autonomia e responsabilidade, já se pode considerar que a mediação foi exitosa, mesmo que não cheguem a uma solução, naquele momento.

Enfim, uma mediação bem-sucedida é aquela em que, promovida eficientemente a facilitação do diálogo pelo mediador e aparadas as arestas, as partes podem retomar a comunicação de forma adequada, passando a conduzir suas relações de forma consensual (SILVA, 2013, p.44).

Com o uso da mediação, o cidadão recupera sua independência e o c ontrole de sua vida pessoal, social e produtiva, num convívio mais racional, adulto e pacífico, trazendo a necessária liberdade e paz social que todos merecemos (SILVA, 2004, p. 13).

Portanto, na mediação se procura rever o conflito positivamente, motivando a autonomia das partes para a produção da diferença, ascende à responsabilidade quanto à tomada 


\title{
RESGATE DA CIDADANIA E DA CULTURA DE PAZ NA TERAPIA
} DO REENCONTRO MEDIADO

de decisão em relação ao conflito, gerando reparações e transformações, configurando uma melhora na qualidade de vida, e conseguinte, paz social.

\section{A TERAPIA DO REENCONTRO MEDIADO}

A mediação vem para constituir uma nova cultura do respeito, da harmonia, do conhecimento, do diálogo e, principalmente, da solidariedade, uma virtude das comunidades que necessita ser resgatada. A solidariedade anda de mãos dadas com a alteridade, se é solidário quando se coloca ao lado do outro para sentir o que o outro sente e compreender o juízo que faz das coisas. A alteridade é o que o ser humano "tem em comum com tudo o que existe e a distinção, que ele partilha com tudo o que vive, tornam-se singularidade, e a pluralidade é a paradoxal pluralidade de seres singulares” (ARENDT, 1977, p. 189).

A terapia do reencontro mediado é a mediação voltada para desenvolver a sensibilidade e o reconhecimento da outridade, é o encontro com o outro, no qual as pessoas dão a si próprias a oportunidade de saírem individualmente de sua zona de conforto para reconstruir espaços para que o reconhecimento se faça presente.

\begin{abstract}
a mediação como uma terapia do amor ou terapia do reencontro mediado.[..] tem como objetivo mediar conflitos a partir da psicoterapia e de um vínculo que se rompeu/perdeu, ou seja, a mediação propicia a inclusão do amor diante do conflito. Além disso, busca-se resgatar a autonomia das partes, gerando uma oportunidade de evolução para os mediados, espaços de encontros. Espaços de transformação e, portanto, de reconhecimento da identidade dos mediados. Esse modelo pode promover, assim uma opção intercultural de mediação e uma forma sensível de promover encontros entre diferentes, já que é baseado na tolerância, no reconhecimento e na compaixão para com o outro. (Warat, 2001, p. 65).
\end{abstract}

Para que possamos olhar o outro, permitindo que o mesmo possa aprender a renascer como ponto de apoio para que haja o reconhecimento e encontros que façam referências a tudo aquilo que a alma busca, para que possamos ver o "rosto secreto de Deus (mediação) ${ }^{3}$ como diferença na resolução de conflitos.

É um processo de sensibilidade, a qual procurar rever os conflitos a partir dos sentimentos, rejeitando o valor da conflitividade, é uma forma de atingir a simplicidade do conflito

A mediação que aponta a sensibilidade, com a ajuda do mediador, procura que as partes deixem sentir o conflito a partir dos seus egos. Tenta que as partes sintam o

\footnotetext{
${ }^{3}$ WARAT - chama a mediação do rosto secreto de Deus. (2010, p. 07). WARAT, Luis Alberto. A rua grita Dionísio. Rio de Janeiro: Lumen Juris, 2010.
} 
conflito tendo por referência os sentimentos que guardam em suas reservas selvagens. O ego e a mente tornam amargurados e violentos os conflitos (Warat, 2001, 39).

Para Warat (2010, p. 3) na mediação não existem regras ou teorias que valham mais que a experiência e os sentimentos dos envolvidos no conflito, assim nenhum mediador, pela teoria waratiana, deve usar uma sessão de mediação como seu espaço de experimentos, como um laboratório a fim de testar a funcionalidade de suas teorias, ainda mais que vão de encontro ou nem mesmo abranjam o conflito que problematiza as partes.

A terapia do reencontro mediador visa a emancipação do indivíduo, “emancipar é conquistar" a partir do momento que observo o mundo e de sua compreensão social com a possibilidade sistêmica, aceitando a articulação dos entre-nos, racionalizando o intercambio comunicativo de encontros compartilhado com a comunidade.

Acredito que o meu fio condutor foi o amor. Ter sabedoria para situar o amor em nossa via ter o aprendizado necessário e esquecido para poder tentar situar no amor, requer um trabalho de auto-conhecimento e maturação pessoal, para não confudi-lo com a dominação, anulação, o submetimento ou qualquer outra forma de dependência (Warat, 2001, p. 67).

A preocupação da terapia do reencontro mediado é com a outridade, ou seja, se preocupa em entender o outro de forma a valorizar as suas razões, a humanização pela pratica da cidadania, como experimento para viver melhor. No litigio não se tem busca a T.RM, visto que a voz do indivíduo é silenciada, por uma petição proposta pelo advogado, que firma um pedido da parte. Ali as pessoas simplesmente acatam a decisão do Juiz, sem ao menos questionar ou resolver os conflitos que se instalam. No processo sempre há um vencedor e um perdedor.

Na mediação os indivíduos constroem a solução para o conflito a partir do olhar do outro e pela sensibilidade de compreender e romper definitivamente com a cultura da indiferença.

É uma aventura do ser, e não de ser pensado como ente. É a aventura de um ser em tensão consigo mesmo que se encontra na aventura de si mesmo, realizando, na relação com o outro, a racionalidade de sua piquê. É um ser que intenta a aventura de se encontrar, na ruptura da indiferença e no estar de um para o outro(Warat, 2001, p. 200).

Segundo Bertaso e Prado (2016, p.57) A prática da comunicação construtiva possui, assim, duas conotações, sendo elas: a positiva e a escuta ativa. A conotação positiva diz respeito ao acolhimento do outro por meio de uma linguagem apreciativa. Já na escuta ativa, há o questionamento sem 


\section{RESGATE DA CIDADANIA E DA CULTURA DE PAZ NA TERAPIA}

DO REENCONTRO MEDIADO

$\overline{\text { julgamento, a busca do reconhecimento e das diferenças, modo especial, }}$ reconhecer o outro.

Importante salientar que a TRM busca-se se chegar ao outro de forma a introduzir um novo sentido ao conflito, na procura de soluções a um procedimento cooperativo, solidário no sentido de promover o afloramento da sensibilidade, racionalidade, compreensão na atuação das divergências. Warat (2001, p. 93) propõem ainda, "a solução de um conflito, a única possível, está em sua alquimia”, significa que a mediação como terapia é uma forma positiva de intervenção no conflito a alteridade e outridade como transformação pela sensibilidade e pelo amor.

A mediação é transformadora pela terapia do reencontro mediado, pois trabalha 0 conflito em todas as suas dimensões restabelecendo dessa forma as relações futuras entre os envolvidos, dando-lhes autonomia para a respectiva solução. Na mediação transformadora, não se busca o acordo, pois o terceiro que aparece no evento denominado mediador, tem a função de criar espaços para que os indivíduos possam se olhar, olhar para si mesmos e para a seu interior buscando a origem e causas desse conflito. Cria encontros em um contexto pedagógico como salienta Warat (2001,p. 136) ser um terceiro que ajuda as pessoas, [...] a um terapeuta deve buscar e encontrar caminhos de solução para os problemas que ali se instalaram.

A mediação se fortalece o compromisso harmonioso com a comunicação participativa, superando obstáculos e proporcionando ações colaborativas na luta e na conquista de leis eficazes, além de fomentar um estimulo ao desenvolvimento de comportamentos conscientes, de um modo geral poderia dizer que mediando se melhora a qualidade de vida (WARAT, 1999, p. 8).

Ainda, segundo Warat a mediação pode ser vista como um componente estruturante da visão ecológica do mundo, um componente estrutural do paradigma político e jurídico da transmodernidade (1999, p. 6).

Dentro disso, a intenção que se extrai com a aplicação da mediação é aproximar o direito a todos os indivíduos, incluindo-os na participação da resolução dos conflitos, tomando para si a responsabilidade de cumprir o que foi estabelecido, apontando para a criação de legislações que preencham os vazios deixados pelo legislador, buscando além formas de solução, a prevenção das contendas. 
O mediador (terapeuta) tem a função de administrar o conflito criando climas de encontro, "sessões nas quais as pessoas possam ressignificar, em corpo e ação na solução" (2001, p. 142) afetiva pela busca da cidadania e da ética.

A terapia do reencontro mediado busca a transformação e compreensão enquanto indivíduo nas suas relações com outro. É o encontro da subjetividade de sustentar, inspirar e compartilhar uma qualidade de vida pela solidariedade, cidadania e democracia nas representações e afetos necessários, com comprometimento e com determinadas renuncias e aceitações para o idealizado e o que pode ser vivido.

\section{O RESGATE DA CIDADANIA E DA CULTURA DE PAZ}

Em razão da crise que o estado vive em decorrência da própria evolução cultural da sociedade com um aumento vertiginoso das demandas judiciais, a insatisfação com a ineficiência jurisdicional estatal, a morosidade dos processos, seu custo e a complexidade das relações, altamente fragilizadas, é urgente a necessidade de que outras formas de solução de disputas promovam uma mudança de racionalidade dos indivíduos.

O conflito é inerente a vida humana e contemporaneamente, é imprescindível analisálo sob uma abordagem relativa à autonomia do indivíduo e sua capacidade de gerir e tratar seus próprios anseios por meio de mecanismos aptos ao desenvolvimento de consensos, restabelecendo um cenário de convívio harmônico em sociedade.

A mediação é sem dúvida um emergente paradigma de solução e prevenção de conflitos, voltada a incluir os cidadãos na participação da solução do conflito, possibilitando uma melhora na qualidade de vida, pois alcança o grau de satisfação nas relações familiares, sociais, amorosas, estética e ambiental. Além de alcançar, por conseguinte a paz social.

Vale destacar que tal consenso deve ser idealizado e construído pelas pessoas, por meio do amor, da sensibilidade, do diálogo e de uma comunicação que atenda o reconhecimento do outro. É preciso sair das prisões do imaginário para encontrar-se consigo mesmo, para encontrar-se com os outros, para encontrar-se com o mundo (WARAT, 2004, p. 43).

Todo ser humano busca o reconhecimento como indivíduo, porém, o desafio de respeitar as diferenças e de integrá-las na sociedade é grande. Embora a mediação busque a pacificação social, ela vai além, busca tratar o sentimento de cada pessoa envolvida, proporcionando a tão almejada qualidade de vida. 


\section{RESGATE DA CIDADANIA E DA CULTURA DE PAZ NA TERAPIA} DO REENCONTRO MEDIADO

É um processo de sensibilidade, a qual procura rever os conflitos a partir de sentimentos, rejeitando o valor da conflitividade interior, é uma forma de atingir a simplicidade do conflito.

As diferentes relações sociais, desde as mais complexas, de interesse econômico e passando pelas relações de valor social, atingem a sociedade de forma contundente, nessa linha, a mediação tem se apresentado como alternativa viável e reconstrutora à propagação da cidadania e da dignidade humana, proporcionando uma maior inclusão social através da prevenção, solução e pacificação de conflitos. Como bem afirma Sales:

[...] através da atividade de mediação, podem ser percebidos mais evidentemente quatro objetivos: solução dos problemas (pela visão positiva de conflito e participação ativa das partes via diálogo, configurando a responsabilidade pela solução), prevenção de conflitos, inclusão social (conscientização de direitos, acesso à justiça) e paz social (2004, p. 27).

Como se vê, o tratamento de conflitos é o objetivo mais claro da mediação, uma vez que se dá através da autonomia das pessoas e do diálogo, no qual os envolvidos em uma conflitividade interagem em busca de uma solução satisfatória para ambos, e isso considerando a multiplicidade da questão que se busca solucionar.

A interação e comunicação entre os envolvidos no conflito são de fundamental importância para a solução que se almeja, pois ambos devem reconhecer a diferença do outro e a autonomia que possuem para gerir e buscar uma composição mais adequada.

Fomenta-se a explanação de Warat:

[...] O acordo decorrente de uma mediação, satisfaz em melhores condições, as necessidades e os desejos das partes, já que estas podem reclamar o que verdadeiramente precisam e não o que a lei lhes reconheceria [...] (1998, p.99).

Um segundo objetivo da mediação é a prevenção de conflitos, uma vez que evita a má administração do mesmo e procura tratá-lo, possibilitando o encontro e a solução, como bem ensina Sales:

\footnotetext{
“a mediação estimula a prevenção da má administração do conflito, pois incentiva: a avaliação das responsabilidades de cada um naquele momento (evitando a atribuição de culpas); a conscientização de adequação das atitudes, dos direitos e deveres e da participação de cada indivíduo para a concretização desses direitos e para a mudanças desses comportamentos; a transformação da visão negativa para a visão positiva dos conflitos (percepção do momento do conflito como oportunidade para o crescimento pessoal e aprimoramento da relação); e, finalmente, o incentivo ao diálogo, possibilitando a comunicação pacífica entre as partes, criando uma cultura do
} 
"encontro por meio da fala", facilitando a obtenção e o cumprimento de possíveis acordos” (2007, p. 36).

Como terceiro objetivo, a mediação é inclusão social, pois a partir dela ocorre uma maior reflexão dos direitos e deveres, dando maior participação dos indivíduos de escolher e decidir o melhor caminho a ser tomado, com consciência e bem estar para os envolvidos. As partes participam efetivamente, sentem-se valorizadas e incluídas.

As pessoas envolvidas no conflito são percebidas como as mais importantes no processo de mediação. Elas são ouvidas, respeitadas, valorizadas e responsáveis pela decisão (SALES, 2007, p. 37).

Assim, esse mecanismo encontra na autonomia o melhor caminho para o evento inquietante, da mesma forma, o conceito de exclusão social é deixado de lado, resultando em uma maior valorização e inclusão do indivíduo na análise e solução do conflito, ante aos documentos e formalidades.

A mediação apresenta-se como uma forma ecológica de resolução de conflitos sociais e de satisfação do desejo, possuindo, nítido caráter pacificador, além de proporcionar celeridade, diminuição de custos e redução de desgastes emocionais.

Para Warat, os caminhos da mediação podem ajudar a recuperar os sentimentos que fazem o que somos, a desfazer-nos das camadas superficiais para sermos muito mais íntegros nos confrontos com o outro (2004, p. 28).

Com isso, se alcança a melhora na qualidade de vida, demonstrando os pontos de vista de cada parte envolvida, reconhecendo o outro e (res) estabelece o diálogo aberto, facilitando o bom relacionamento não só na solução do conflito, mas uma sadia convivência entre as pessoas.

Como bem expressa Sales:

Ensina-se a paz quando se resolve e se previne a má administração dos conflitos;
quando se busca o diálogo; quando se possibilita a discussão sobre direitos e deveres
e sobre responsabilidade social; quando se substitui a competição pela cooperação -
o perde-ganha pelo ganha-ganha (2007, p. 38)

Dessa feita, a mediação é instrumento apropriado ao tratamento e solução dos conflitos, pois reflete diretamente na qualidade de vida dos envolvidos, trazendo a paz social em âmbito local e mundial. 


\section{RESGATE DA CIDADANIA E DA CULTURA DE PAZ NA TERAPIA}

DO REENCONTRO MEDIADO

A mediação trata-se de um procedimento extremamente humano, pois quando as partes são estimuladas a solucionarem conjuntamente os seus conflitos, incentiva a cooperação e a solidariedade entre ambas, que tem a oportunidade de aprender e atentar para o que a outra tem a dizer.

Trata-se de uma visão de mundo pautado num critério ecológico e epistêmico de sentido, de forma a conscientizar os indivíduos de seus direitos e deveres e a prevenir a violência. E quando as partes estão dispostas a transformarem-se em busca de um equilíbrio, a sociedade também se transforma e assim se ascende à pacificação social.

A melhora na qualidade de vida e paz social somente se é alcançada quando as pessoas buscam um encontro consigo mesmo. Então, [...] mediação nada mais é que viver, viver em harmonia com a própria interioridade e com os outros, viver em harmonia com a própria reserva selvagem (WARAT, 2004, p. 28).

Como se vê, a mediação propõe efeitos para a melhora na qualidade de vida e meio para a pacificação social, pois está eivada na autonomia dos indivíduos, ou seja, no empoderamento das partes para a gerência e solução de seus conflitos cotidianos, conscientizando-os de sua responsabilidade pelos seus atos conflitivos e estabelece a cultura da paz a partir do momento do olhar do outro e da solidariedade.

\section{CONSIDERAÇÕES FINAIS}

Diante do que foi exposto pode-se salientar que a mediação é sentimento, é outridade é diferença, é inconformismo, fonte de cidadania. Através da mediação há a capacidade de reencontro e de união e é através da união e dos debates com a diferença e com e que é possível formar um novo movimento social emancipador, solidário, humanista a constituir a visibilidade de entendimento entre os indivíduos.

A mediação nos momentos de discussão entre movimentos sociais e poderes constituídos pode referenciar a realização da cidadania, enquanto reconhecimento, gerando condições e possibilidades do Estado e sua população, enquanto diversidade, prosperar e fazer valer o direito a cidadania para todos

A operacionalização desse meio de tratamento de conflitos possibilita as próprias partes construírem suas respostas livremente. Entretanto, é possível ainda concluir que na mediação não se busca apenas acabar com o litígio, mas sim com a causa (origem) do mesmo, 
cujas soluções são fundadas no respeito ao outro e no benefício mútuo, a partir da avaliação interna e pessoal de cada indivíduo.

Sob essa ótica, é importante reconhecer que por meio da mediação verifica-se a ressignificação do conflito e a promoção da autonomia das partes, a partir da análise de suas próprias representações. Além disso, perfaz a construção de uma justiça mais humanizada e a consequente satisfação dos desejos humanos.

Na perspectiva investigada, observou-se que a mediação é um instrumento apto a propiciar um diálogo que possa atrair a inclusão e a participação dos indivíduos para a solução do conflito, bem como, alinha a igualdade a partir do reconhecimento do outro.

Por fim, a contribuição da mediação por meio da terapia do reencontro mediado é o meio de se chegar a cidadania, valorizando e consolidando o diálogo não violento, o qual é capaz de relacionar nossos sentimentos às próprias necessidades, protegendo os indivíduos de forma comprometida com a outridade transformando e reconhecendo a igualdade e diferença no interior dos conflitos, como forma de cultura da paz e resgate da cidadania. 


\section{REFERENCIAS}

ARENDT, Hannah. A condição humana. Rio de Janeiro: Forense Universitária, 1997.

BERTASO, João M. PRADO, Keila Sim do. Aspectos da mediação comunitária, cidadania e democracia. Disponível em: www.univali.br/periodicos, acesso em 26 de mar.2018

SALES, Lília Maia de Morais. Mediação de Conflitos. Florianópolis: Conceito Editorial, 2007.

Justiça e Mediação de Conflitos. Belo Horizonte: DelRey, 2004.

SILVA, João Roberto. A Mediação e o Processo de Mediação. São Paulo, 2004.

SILVA, Adonias Osias da; ARAÚJO, Carla Regina de Freitas. Mediação como instrumento para Justiça da Paz. Revista Científica Multidisciplinar Núcleo do Conhecimento. Vol. 1. Ano. 1. Março. 2016, pp : 21- 39 - ISSN: 2448-0959. Disponível em https://www.nucleodoconhecimento.com.br/lei/mediacao-como-instrumento-para-justica-dapaz Acesso em 08/12/2013.

WARAT, Luis Alberto. Em Nome do Acordo, Florianópolis: Almed, 2010.

. Em Nome do Acordo: A Mediação no Direito. Argentina: Almed, 1999.

. Em Nome do Acordo: A Mediação no Direito. Argentina: Almed, 1998.

. O Ofício do Mediador, Florianópolis, Editora Habitus, 2001.

. A Rua Grita Dionísio: Direitos Humanos da Alteridade, Surrealismo e Cartografia.

Florianópolis: Lumen Juris, 2010.

. A Epistemologia e o Ensino do Direito: O sonho acabou. Vol. II. Coordenadores:

Orides Mezzaroba, Arno Dal Ri Junior, Aires José Rover, Cláudia Servilha Monteiro. Florianópolis: Fundação Boiteux, 2004. 